\title{
Structure of University Database System and Data Analysis
}

\author{
Masaaki Ida* \\ *National Institution for Academic Degrees and University Evaluation, 1-29-1 Gakuen-nishimachi, Kodaira, Tokyo, Japan \\ ida@niad.ac.jp
}

\begin{abstract}
This paper describes the state of research and development of data structure for university data, especially, Japanese university basic survey data and its application for business intelligence analysis. Our research and development are aimed for university data reporting and comparative analysis on the data with consideration on general university information structure. Institutional data of university, college, or college of technology are substantially important for data analysis or knowledge discovery in the higher education management field. However, university institutional data are not necessarily standardized and compiled, so it is difficult to integrate their information for various reporting and data analysis. In the past decade, a number of investigations of the integrated university database have been done to deal with various kinds of university institutional information including university survey or school basic survey data by considering the structured university data. This paper describes the state of our research and development of structure of university database and data analysis system, which are effectively utilized for data reporting and university comparative analysis. We explain (i) development of various data reporting and analysis system of university database system, and (ii) proposal of generalized layered university database structure based on Link-based data set.
\end{abstract}

Keywords - Business intelligence, Data structure, Link base, School basic survey, XBRL.

\section{INTRODUCTION}

\section{A. University Institutional Data}

Development of education-related databases is substantially important for data analysis and knowledge discovery in education field [1]. Institutional data of universities, such as the number of various kinds of academic staffs, are difficult to analyze since they are not necessarily fully standardized and integrated in each university itself or in national level education-related agencies. However, some advanced university database systems are progressively developing.

In the United States, Integrated Postsecondary Education Data System (IPEDS) [2] of National Center for Education Statistics (NCES) has been developed to collect and analyze basic institutional information about universities and colleges in U.S. The system standardizes and accumulates these information nationwide. This system comprehensively holds basic institutional data, such as institutional characteristic, degree completion, enrollment, human resource, finance, student financial aid, graduation rate, and so on. Moreover, this system is equipped with facilitated data analysis tools to conduct university comparative analysis.

In Japan, Ministry of Education, Culture, Sports, Science and Technology (MEXT) collects basic information about higher education institutions in Japan [3],[4]. This basic survey data include the yearly information of higher education institutions, such as the number of faculties or staffs, enrolled students by grade (undergraduate, graduate, foreign student), graduates by subsequent course, students those who were employed after graduation by industry and by occupation, academic faculties, and facilities, financial data and so on.

Figure 1 shows two examples of the statistics of university basic survey for all national and prefectural universities in Japan [5].

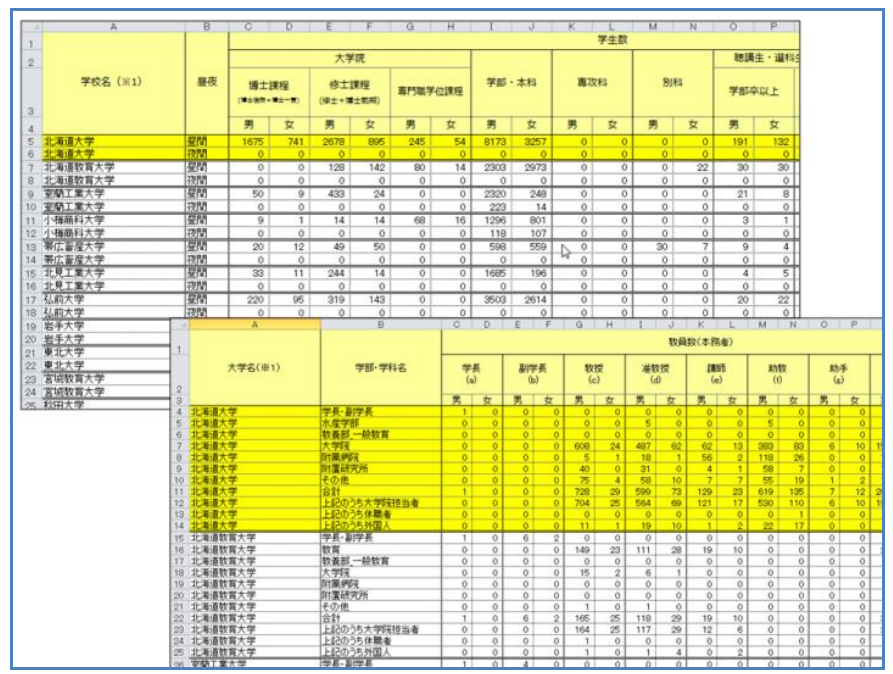

Figure 1. University elementary data (number of students, and number of academic faculty members)

\section{B. eXtensible Business Reporting Language}

The eXtensible Business Reporting Language (XBRL) is one of the computer languages based on XML, which is a standard for the electronic exchange of data between businesses on the internet [6]--[8]. XBRL utilizes some XML technologies such as XML Schema and XLink standards. Based on XML, tags are applied to items of financial business data so that financial data can be processed efficiently by computer software. XBRL 
is implemented in a wide range of scenes such as tax payment system and financial data transfer system in stock exchange.

XBRL consists of XBRL Report Instance, containing primarily the business facts being reported, and a set of Taxonomies, defining metadata about these facts, such as what the facts mean and how they relate to one another (Figure 2):

- Instance holds the following information: business facts, contexts (date and time information, scenario), units, footnote, and references.

- Taxonomies are the reporting-area specific hierarchical dictionaries. The XBRL specification defines five different kinds of linkbases (Label linkbase, Reference linkbase, Definition linkbase, Calculation linkbase, and Presentation linkbase). Taxonomies consist of hierarchical structure ((1) standard taxonomy, (2) industry taxonomy, (3) company taxonomy).

Instance and company taxonomy of financial reports must be submitted from individual company to government agency. Different taxonomies are required for different purposes in various application fields.

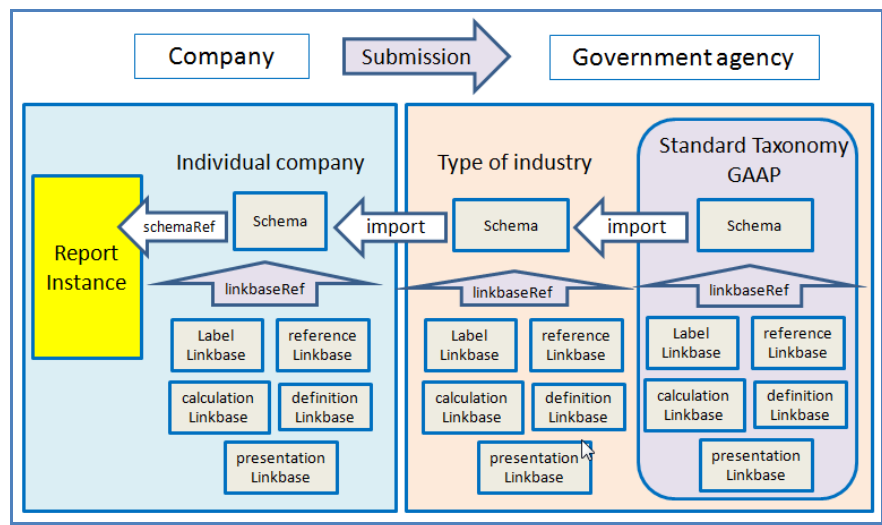

Figure 2. Submission of financial reports from individual company to government agency: XBRL Instance and layered Taxonomies ((1) standard taxonomy, (2) industry taxonomy, (3) company taxonomy)

In this paper, we utilize the technology of XBRL (Linkbased data set) for expressing the various kinds of university information. We extend or modify taxonomies of standard XBRL to university information.

\section{DATA WARE HOUSE AND ANALYSIS SYSTEM}

\section{A. Data Flow of University Survey Data for Analysis}

We have been developing our university database and various web services up to now [9]-[11]. Figure 3 shows the overview of our research and development of the data reporting and analysis system based on our university database. On the left side of the figure, various data (university survey data, other university related data, code and so on) in the various kind of data system and media (relational database, XML database, Excel and so on) are integrated into the central database system or data warehouse (DWH) with Extract/Transform/Load (ETL) tools. Compiled university data in DWH are transformed into data tables or charts in Excel sheets, PDF files or web pages, or transformed into the cubes in OLAP analysis server in more sophisticated and layered data form.

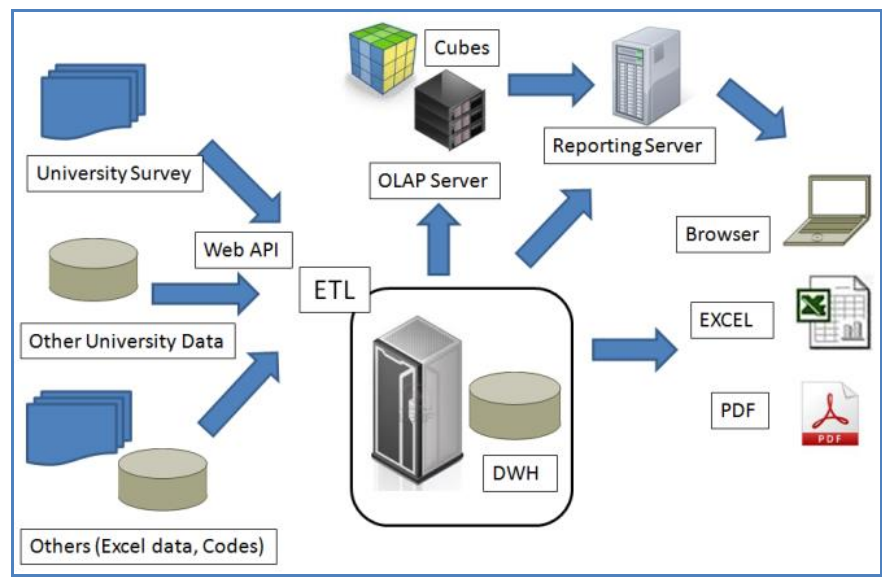

Figure 3. University database and analysis system

\section{B. Reporting System}

Receiving the university data form DWH or OLAP server, several type of university analysis repots are generated in the form of Excel or PDF files, or more interactive reporting web system (server) in the right upper side of Figure 3. Figure 4 shows an example of repots in Excel sheet, and Figure 5 shows examples of interactive report web page (tables and charts) on the reporting web server.

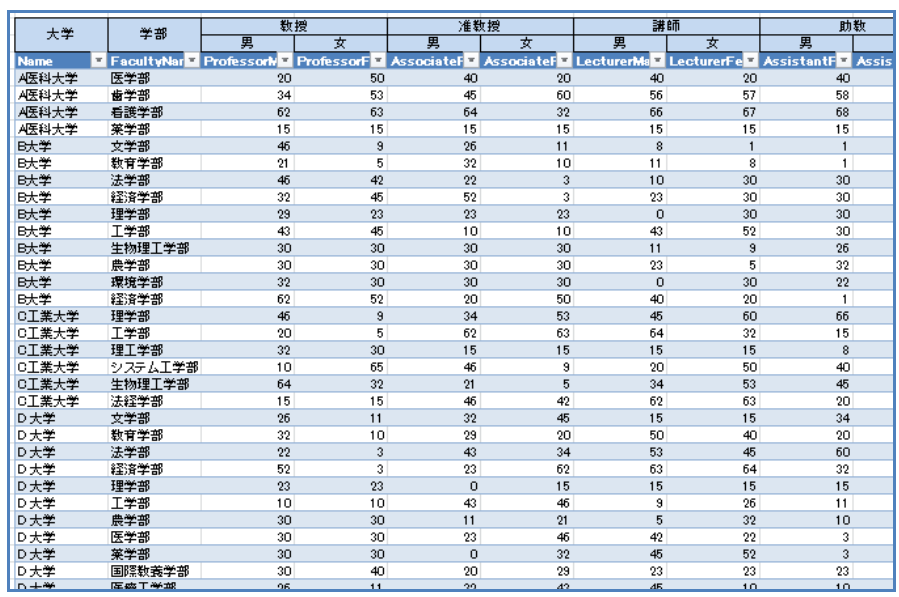

Figure 4. University analysis report results in Excel sheet (universities and the numbers of academic faculties: professor, associate professor, lecturer; male and female) 


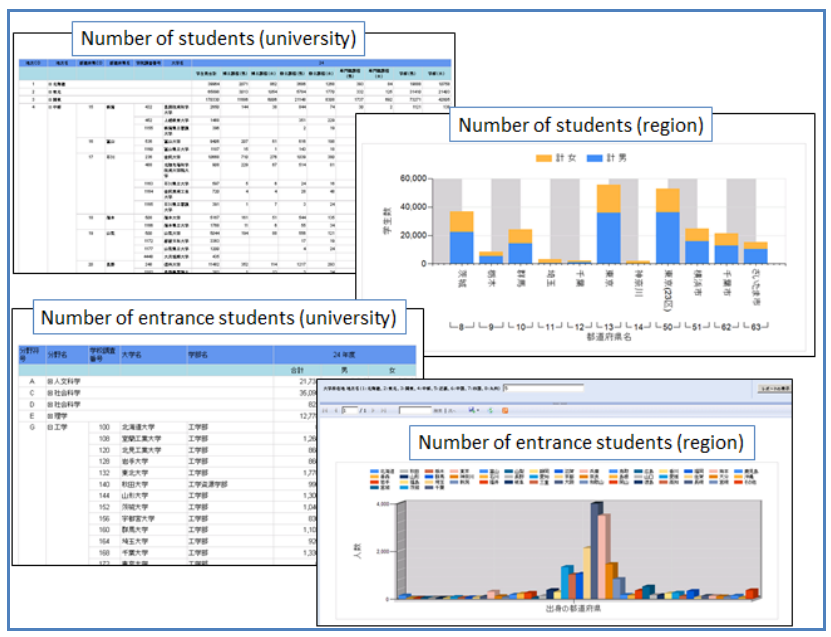

Figure 5. Web based reporting system (number of students for universities and number of students for regions)

\section{Comparative Analysis}

We have also developed university data analysis system utilizing our web services and analysis server (cube, OLAP server) for university comparative analysis in the upper side of the Figure 3. In this section we show some result examples of comparative analysis using data derived from the services.

We compare several universities in terms of similar university characteristics. Some analysis and reporting tools with confidential university data (university basic survey data) and graphical tools are utilized. As shown in Figure 6 and 7, several convenient manual operations for selection of elements or setting of graphical representation are available with friendly user interface. Database connections to "universe" or "cubes" of OLAP server are easily realized. Moreover, setting of calculation formula (summation, average, median, various indicators) is easy to operate. Table and cross table, and graph generations, and report output in Excel or PDF format are relatively easy with interactive analysis tools (filtering, drill down methods). Figure 6 and 7 show some analysis results produced by using cubes and analysis server. Figure 8 shows an analysis results combined with Excel analysis tool (Power Pivot).

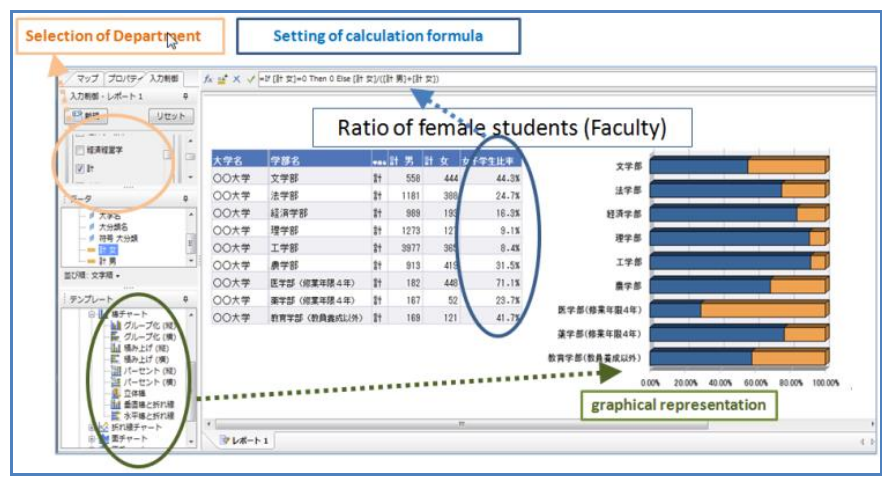

Figure 6. Analysis result 1 (graphical representation) with OLAP cube for the number of female students

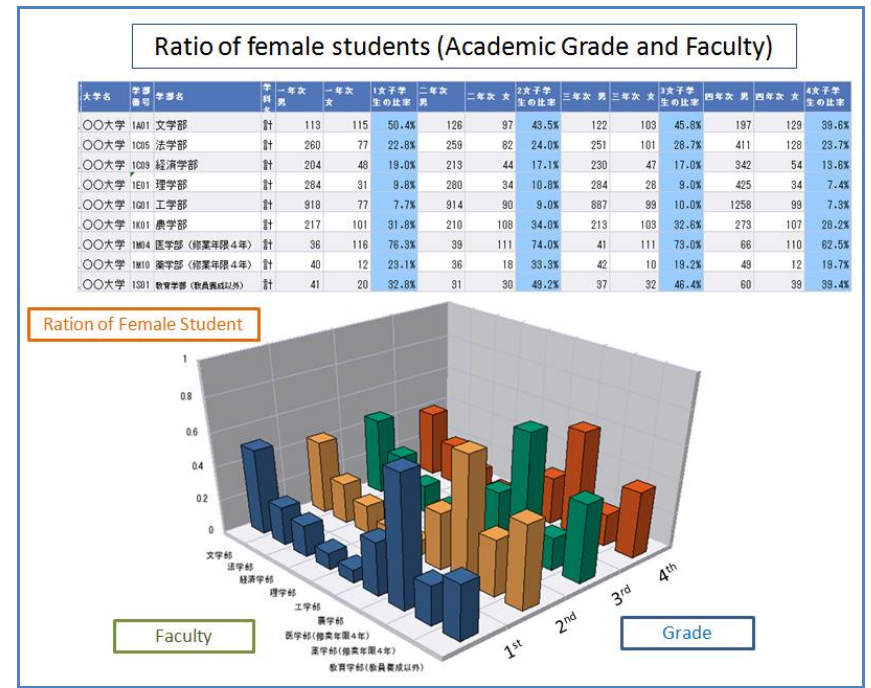

Figure 7. Analysis result 2 (graphical representation) with OLAP cube

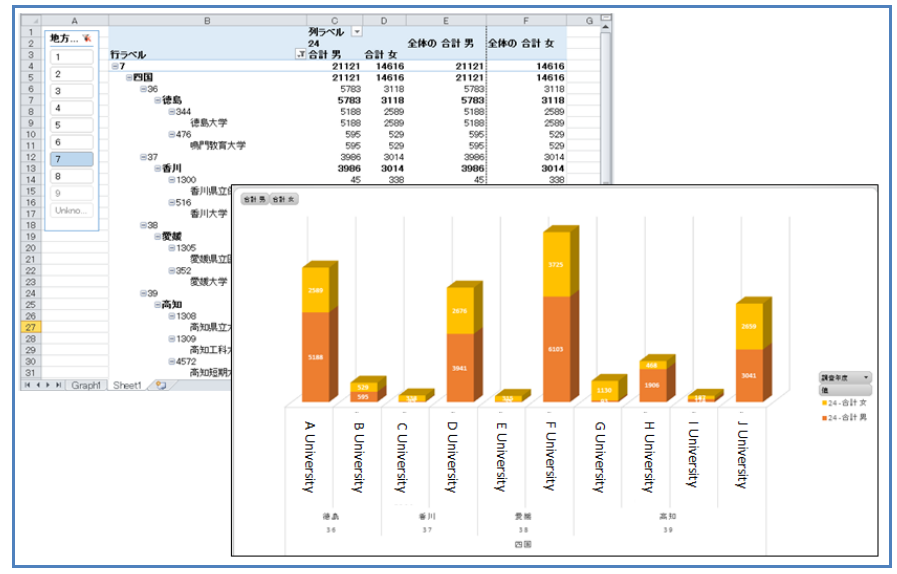

Figure 8. Analysis result 3 combined with Excel analysis tool (Power Pivot) of selections for region and fiscal year

We come to be able to deal with complex university data in a convenient manner. However, in order to deal with more complex and diverse university data, we have to deal with more general and hierarchical data structure described in XML type language.

\section{UNIVERSITY DATABASE AND XBRL EXTENSION}

\section{A. University Database System}

In order to deal with more general reporting and analysis case, we propose a layered structure of university database system based on the link-based data sets. Figure 9 shows the layered university information system based on XBRL. As explained previous section, we have developed DWH, cubes (OLAP system), and analysis (report) system which are described in the lower side of the figure. In order to deal with more complex and diverse university data, we proposed the eXtensible Education Report Language (XERL) based on XBRL as shown in the upper side of the figure. The scope of this language is a generalization, or extension, from financial data of XBRL to university survey data such as university financial data, students, academic faculty, staff, and other 
university-related data. This generalization can be achieved by the extended taxonomy design of XERL for university data.

In Figure 9, data flows and operations of (1) and (2) were explained in the previous section (as Business Intelligence analysis). Proposed data flow and operation of (3) are more general data treatment for generalized university data.

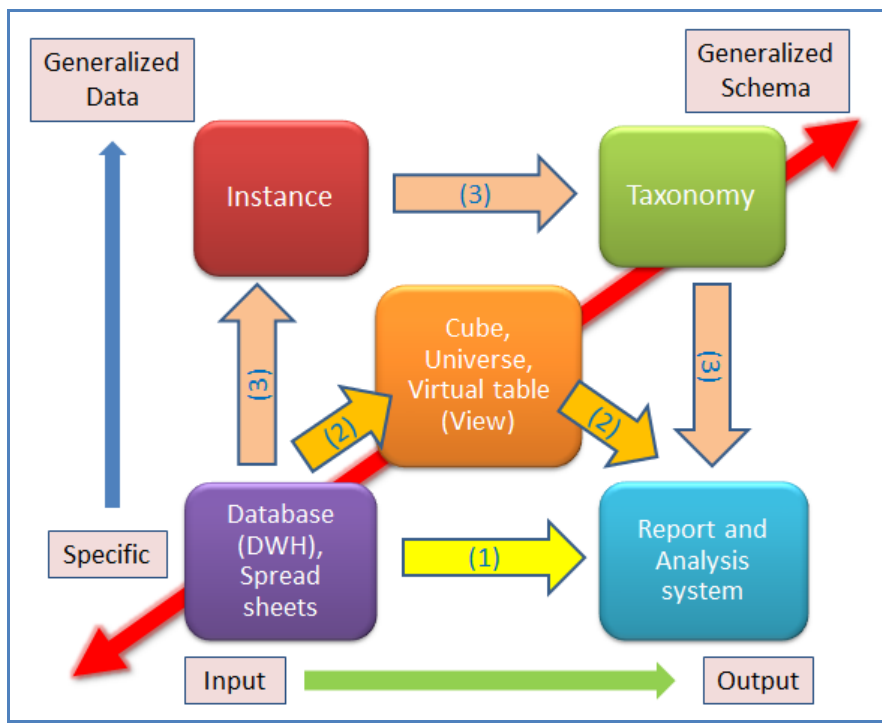

Figure 9. Structure of generarized university database system (specificgeneralized) and data flows (input-output)

\section{B. Concrete Instance Files of University Database System}

Figure 10 shows a part of specific instance file extended from XBRL (e.g. "Books", "Art object and collection" are extensions) as shown in the left upper side of Figure 9 for certain university.

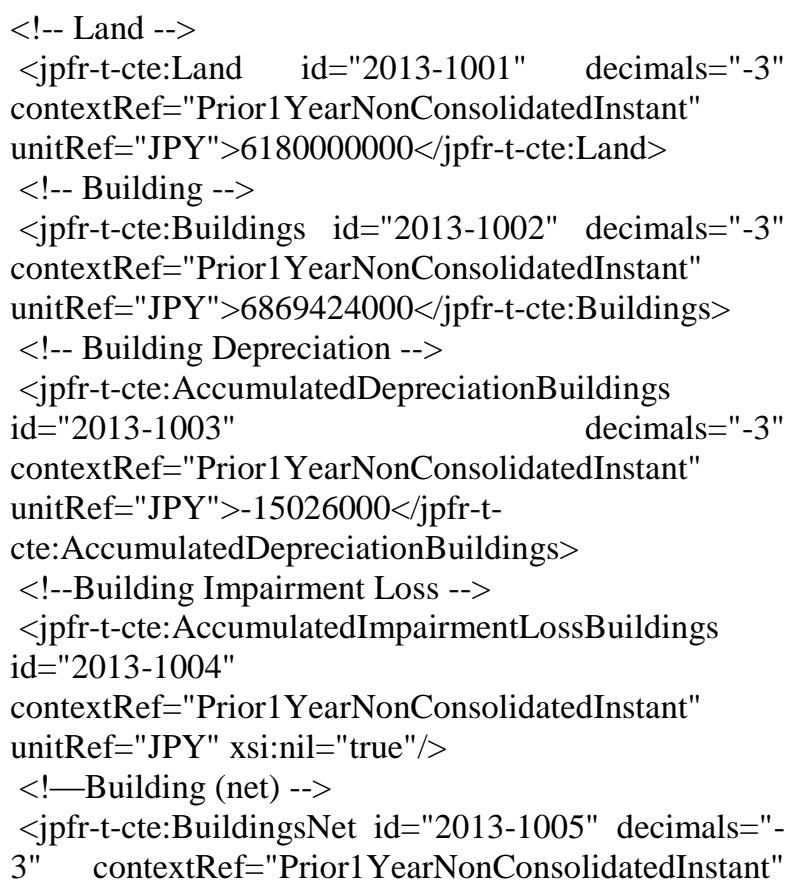

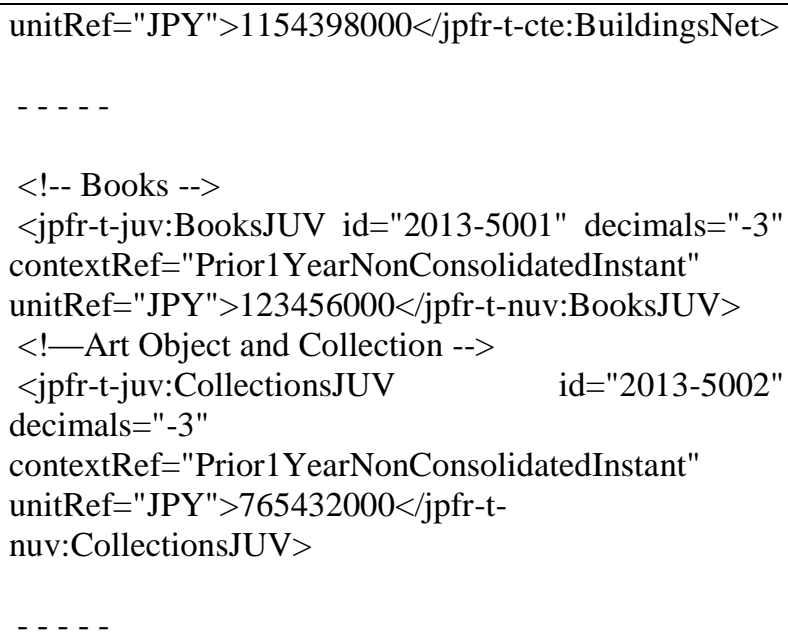

Figure 10. Part of a specific instance file for certain university

Corresponding data relations (data mappings) between contents ((A) relational data tables or Excel sheets, (B) instance XML files, (C) hierarchy schema (taxonomies), (D) analysis tables and charts) of each parts of our generalized university database system are shown in Figure 11.

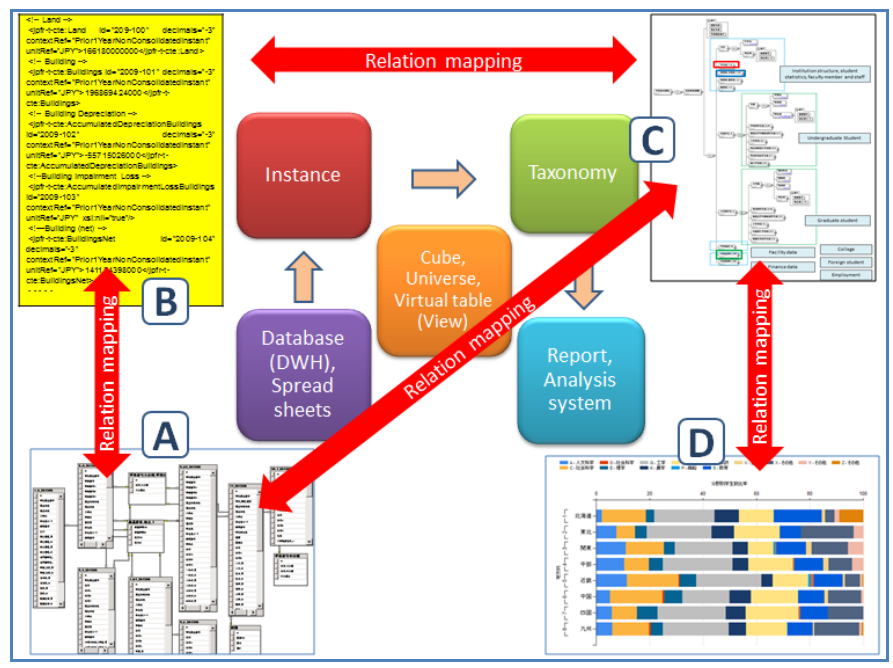

Figure 11. Corresponding relations between contents of each parts of the generalized university database system

\section{CONCLuSIONS}

Applications of education related information are substantially important for data analysis and knowledge discovery in education field. This paper described the state of R \& D of data structure of university survey database system, which is utilized for analysis of university characteristics. In this paper, we described (i) development of various data reporting and analysis system of university database, (ii) proposal of the generalized structure of university database system based on the sets of Link-based data as an extension from XBRL. We examined our extension of taxonomy design on the data for Japanese university basic survey. Moreover, 
we extended XBRL taxonomies that can produce more general institutional indicators for higher education institutions. In order to handle more general university data such as the data between some countries, we have to coordinate differences between those data for effective comparisons by utilizing our link-based data structure. We hope that our proposal will play an important role as an infrastructure for data analysis and knowledge discovery in higher education field.

\section{REFERENCES}

[1] C. Romero, S. Ventura, M. Pechenizkiy and R. Baker (eds.), Handbook of Educational Data Mining, CRC Press, 2010.

[2] NCES, Integrated Postsecondary Education Data System, IPEDS, http://nces.ed.gov/ipeds/

[3] Ministry of Education, Culture, Sports, Science and Technology, basic information of higher education institutions in Japan http://www.mext.go.jp/b menu/toukei/

[4] Example of survey sheet (sheet of "faculty and student"), http://www.mext.go.jp/component/b_menu/other/_icsFiles/afieldfile/20 12/03/30/1318957_3.pdf

[5] Japanese University Basic Information, [Online]. Available: http://portal.niad.ac.jp/ptrt/table.html
[6] eXtensible Business Reporting Language, http://www.xbrl.org/

[7] R. Debreceny, C. Felden, B. Ochocki, M. Piechocki, et al., XBRL for Interactive Data: Engineering the Information Value Chain, Springer, 2009.

[8] C. Hoffmann, and L.A. Watson, XBRL, Wiley, 2010

[9] M. Ida, "Web Service and Visualization for Higher Education Information Providing Service," Proc. of ICSESS2010, pp.415-418, 2010 .

[10] M. Ida, "XBRL Extension for Knowledge Discovery in Higher Education," Proc. of the 8th International Conference on Fuzzy Systems and Knowledge Discovery, pp.2177-2180, 2011.

[11] M. Ida, "XBRL Financial Database for Higher Education Institutions," Proc. of the 14th International Conference on Advanced Communication Technology, pp. 398-401, 2012.

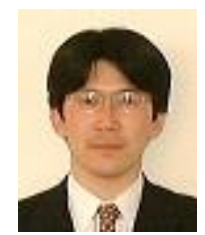

Masaaki Ida was received the B.E., M.E, and Ph.D. degrees in engineering from Kyoto University, Kyoto, Japan. He is currently a professor of National Institution for Academic Degrees and University Evaluation. His current research interests are in computer and systems science, especially in advanced database system and communication technology in higher education. 